\title{
Subconvulsive Dose of Kainic Acid Transiently Increases the Locomotor Activity of Adult Wistar Rats
}

\author{
V. RILJAK ${ }^{1}$, D. MAREŠOVÁ ${ }^{1}$, J. POKORNÝ ${ }^{1}$, K. JANDOVÁ ${ }^{1}$ \\ ${ }^{1}$ Institute of Physiology, First Faculty of Medicine, Charles University in Prague, Prague, Czech \\ Republic
}

Received March 28, 2014

Accepted September 1, 2014

On-line October 15, 2014

\begin{abstract}
Summary
Kainic acid (KA) is a potent neurotoxic substance valuable in research of temporal lobe epilepsy. We tested how subconvulsive dose of KA influences spontaneous behavior of adult Wistar rats. Animals were treated with $5 \mathrm{mg} / \mathrm{kg}$ of $\mathrm{KA}$ and tested in Laboras open field test for one hour in order to evaluate various behavioral parameters. Week after the KA treatment animals were tested again in Laboras open field test. Finally, rat's brains were sliced and stained with Fluoro-Jade $B$ to detect possible neuronal degeneration. Treatment with KA increased the time spent by locomotion $(p<0.01)$, exploratory rearing $(p<0.05)$ and animals traveled longer distance $(p<0.01)$. These parameters tended to increase thirty minutes after KA administration. Week after the treatment we did not found differences in any measured behavioral parameter. Histology in terms of Fluoro-Jade $B$ staining did not reveal any obvious neuronal damage in hippocampus. These results demonstrate that subconvulsive KA dose changes the behavioral parameters only transiently. Clarification of timing of the KA induced changes may contribute to understand mutual relationship between non-convulsive seizures and behavioral/cognitive consequences.
\end{abstract}

\section{Key words}

Kainic acid $\bullet$ Laboras $\bullet$ Behavior $\bullet$ Wistar rat $\bullet$ Locomotor activity

\section{Corresponding author}

V. Riljak, Institute of Physiology, First Faculty of Medicine, Charles University in Prague, Albertov 5, 12800 Prague 2, Czech Republic. E-mail: vladimir.riljak@If1.cuni.cz
Kainic acid (KA) is agonist for kainate subtype receptors of excitatory amino acids (Monaghan and Cotman 1982) and its administration was revealed as a good strategy to model the clinical and neuropathological features of temporal lobe epilepsy (Olney et al. 1974). Most commonly, a systemic administration of KA in convulsive dose $(10 \mathrm{mg} / \mathrm{kg})$ is used to induce status epilepticus leading to massive excitotoxic damage of neuronal tissue (Doble 1999, Riljak et al. 2007). The KA induced status epilepticus is followed by latent period and occurrence of spontaneous recurrent seizures (Albala et al. 1984, Sperk 1994, Drexel et al. 2012). Morphological damage, as a consequence of KA administration is mostly expressed in brain structures rich of kainate receptors like amygdala and hippocampus - degeneration of CA1 and CA3 hippocampal subfields with final hippocampal sclerosis and the mossy fiber sprouting in the inner molecular layer of the dentate gyrus was documented elsewhere (Sperk 1994, Drexel et al. 2012). Mentioned deleterious KA effects are mediated partially by excessive glutamate release, partially by generation free radical species (Doble 1999). On the other hand, systemic administration of $\mathrm{KA}$ in doses lower than $6 \mathrm{mg} / \mathrm{kg}$ induces limbic non-convulsive seizures of variable intensity apparent as scratching and wet dog shakes (Sperk 1994). However behavioral consequences of low dose KA treatment have been described by few authors only (Koryntová and Mareš 1998, Kubová et al. 2001). In order to observe the spontaneous behavior of rat, the open field test is usually used (in many different versions). The time of such studies is intentionally limited (usually ten

PHYSIOLOGICAL RESEARCH • ISSN 0862-8408 (print) • ISSN 1802-9973 (online)

(c) 2015 Institute of Physiology v.v.i., Academy of Sciences of the Czech Republic, Prague, Czech Republic

Fax +420 241062 164, e-mail: physres@biomed.cas.cz, www.biomed.cas.cz/physiolres 
or fifteen minutes). Therefore we decided to use automated observational system LABORAS ${ }^{\mathrm{TM}}$ (Metris B.V., Netherland, in the text referred as Laboras open field test, LOFT), to extend this observation to one hour and to catch even the whole behavioral profile of animals placed into the novel environment.

The focus of current study was to further understand the consequences of low dose KA exposure and to determine, whether KA effect on animal behavior is long-lasting. LOFT allows to classified spectrum of different behavioral parameters and characteristics like locomotion, average speed during test session, duration of immobility, distance traveled by animal and rearing occurrence (Schwarz et al. 2014, Jandova et al. 2013). LOFT took place immediately after KA administration and one week after. Secondly, the immunohistological analysis of hippocampus was made (Fluoro-Jade B) to visualize hippocampal degeneration. We hypothesized, that KA increases the locomotion and average speed of animals, presuming that activation of glutamate receptors could cause excitation and pro-exploratory behavior. Secondly we hypothesized, that behavioral changes caused by low KA dose will have long-lasting effects, as far as recurrent seizures appear.

Experiment was performed in 20 male Wistar rats (purchased from Velaz, Czech Republic). The average weight of animal was $235 \pm 2.0 \mathrm{~g}$ (8 weeks old as they entered the experiment). All animals were housed 4 animals per cage in a temperature-controlled environment $\left(22-23{ }^{\circ} \mathrm{C}\right)$ with free access to food and water on a standard $12 \mathrm{~h}$ light/dark cycles (with lights on at 06:00 h). All experiments took place between 08:00 and 15:00 in a room with lights on (light intensity between 150 and $200 \mathrm{~lx}$ at the level of cages). Animals were weighted prior each session, marked and randomly assigned either to $\mathrm{KA}$ treated group $(5 \mathrm{mg} / \mathrm{kg}$ of kainic acid purchased from Sigma, dissolved in saline and injected i.p.) or to second group treated with saline (i.p.) in equal volume. Animals were then placed and tested in Laboras apparatus (Metris B.V., Netherlands) to detect their spontaneous behavior for one hour. During the tests animals had not access to either food or water. After the period of one week the animals were again tested in LOFT for one hour. Experiment was ended day after the second LOFT - animals were transcardially perfused under deep thiopental anesthesia, brains were removed from the skull and processed for histological examination by Fluoro-Jade B staining (detailed description please see Riljak et al. 2007). All experiments were reviewed and approved by the Institutional Animal Care and Use Committee and are in agreement with the Czech Government Requirements and Requirements of European Communities Council Directive (86/609/EEC).

Each measured behavioral parameter was analyzed separately. Laboras data were subjected to nonparametric tests (Mann-Whitney test). Firstly, results from whole one hour session were analyzed. Then the same data were analyzed separated in ten minutes intervals. To compare the differences between particular groups within $10 \mathrm{~min}$ intervals Mann-Whitney test was used. Results were considered significant if $p<0.05$.

All animals treated with kainic acid (or saline) survived, but the weight of KA animals was lowered $(252.5 \pm 7.92 \mathrm{~g})$ one week after the injection when compared with saline treated rats $(271.1 \pm 5.14 \mathrm{~g}, \mathrm{p}<0.05)$. Immediately after the KA injection animals moved more: they spent $232.1 \pm 47.55 \mathrm{~s}$ in locomotion (saline treated $52.7 \pm 7.15 \mathrm{~s}, \mathrm{p}<0.01)$ and their average speed was higher $(4.852 \pm 1.0 \mathrm{~mm} / \mathrm{s}$ in KA group, saline treated $0.98 \pm 0.15$ $\mathrm{mm} / \mathrm{s}, \mathrm{p}<0.01$ ). Vertical activity (represent as time spent by rearing) was increased as well (KA group $412.7 \pm 47.64 \mathrm{~s}$, saline treated group $251.6 \pm 42.21 \mathrm{~s}$, $\mathrm{p}<0.05$ ). If spontaneous behavior was analyzed over 10 min intervals (Fig. 1), it showed that the immediate effect of KA-treatment on nearly all tested behavioral parameters became significant after thirty minutes (in the $4^{\text {th }}$ analyzed interval for the first time). Proportionally to an increase of locomotor activity, immobility declined and animals tended to explore more even in the vertical sense of direction (rearing). Finally, KA treated rats reached higher average speed during their movement and by this they travelled much longer distance $(17.48 \pm 3.61 \mathrm{~m}$ vs. $3.55 \pm 0.55 \mathrm{~m}, \mathrm{p}<0.01)$ if compared with controls. Week after the KA administration there was no difference in any of tested behavioral parameter. KA treated rats travelled yet longer distance $(7.99 \pm 3.60 \mathrm{~m}$ vs. $3.73 \pm 0.64 \mathrm{~m}$, n.s.) but that difference was statistically insignificant. Histological examination of hippocampal area did not reveal Fluoro-Jade B positive cells.

This study shows detailed description of behavioral changes elicited by systemic application of subconvulsive dose of KA. We demonstrated the capability of Laboras system to generate reasonable and interpretable data that could quantify spontaneous behavior of animals challenged by excitotoxic substance for long period of time. Second important aspect: the setup does not require significant interference of 


\section{Locomotion}

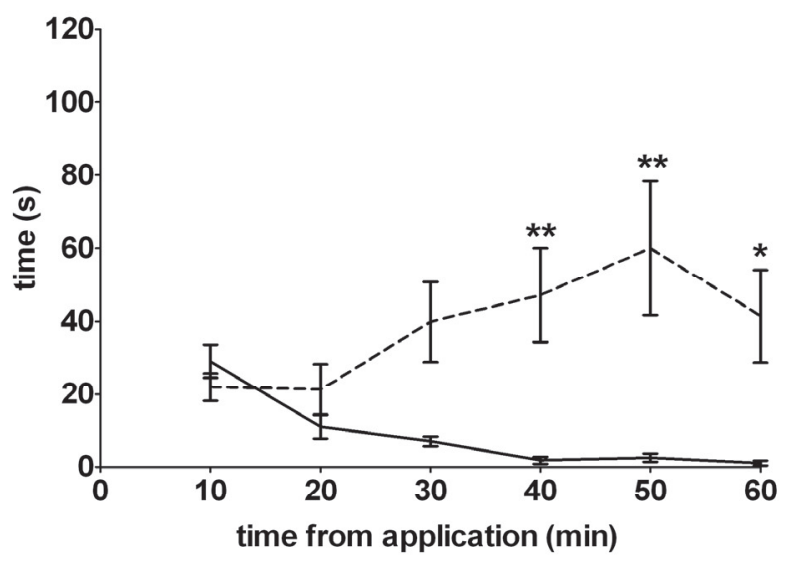

\section{Rearing}

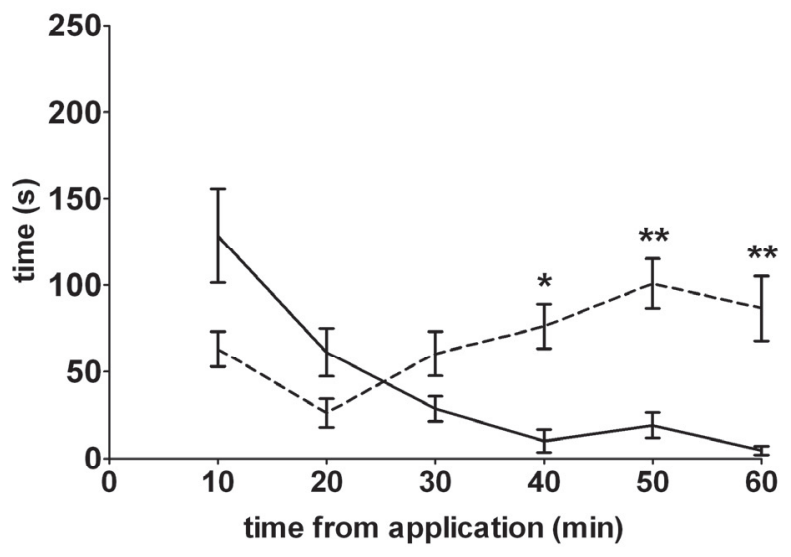

Immobility

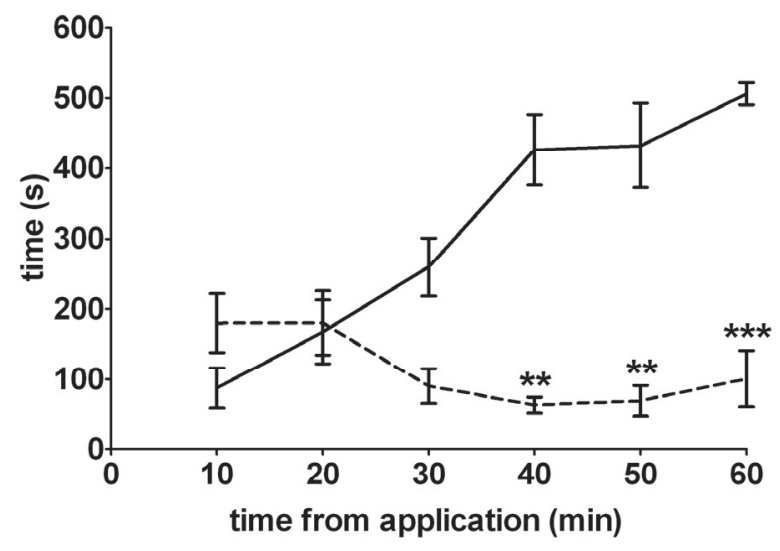

Speed

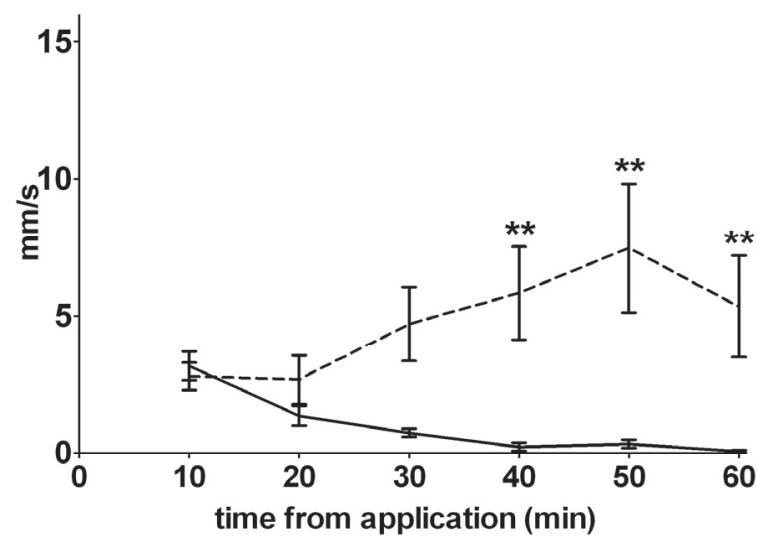

\section{Distance}

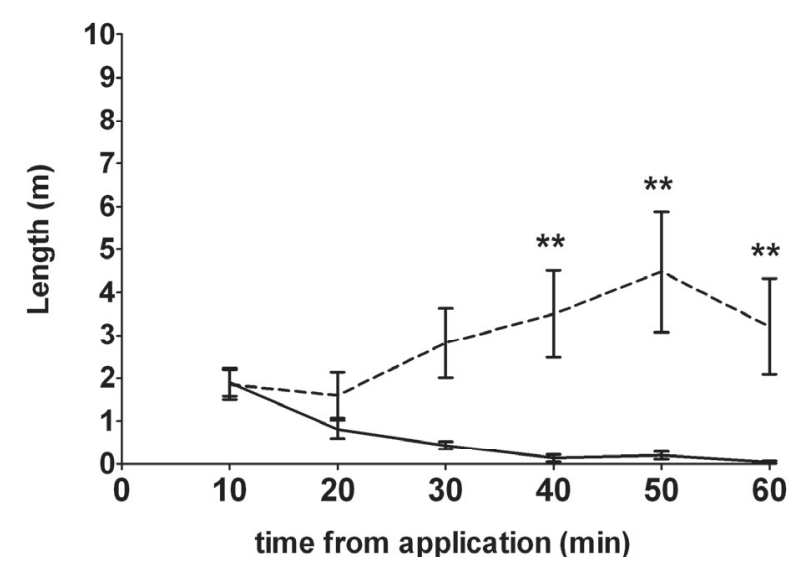

Fig. 1. Acute effects of systemic administration of subconvulsive dose of KA on locomotor activity (duration), immobility (duration), rearing (duration), average speed and distance travelled. Whole one hour session divided into six 10 -min intervals. Solid lines represent saline treated rats, dashed lines kainic acid treated rats. * results significant at $\mathrm{p}<0.05, * *$ results significant at $\mathrm{p}<0.01$, $* * *$ results significant at $\mathrm{p}<0.001$. Error bars were calculated as $\pm \mathrm{SEM}$.

researcher (observation of arena) and might monitor the behavior for days. In this study we were interested how subconvulsive dose of KA influences the immediate and delayed behavioral response of adult rats. Moreover, we were wondering how KA could influence parameters such as average and maximal speed. The effect of KA 
was observed since the $30^{\text {th }}$ min of the experiment, when KA treated animals started to increase their locomotor activity, while controls in this time period begun to habituate to the novel environment. We hypothesize that this relates probably with pharmacokinetics of KA. KA treated animals were also less immobile during the second half of test session (while controls stopped to explore new environment and became immobile). These effects could be ascribed to excitatory effect of kainic acid and because animals in our experiment never showed severe tonic-clonic convulsions or lost of posture control so their mobility (and possibility to explore) remained spared. These findings are in line with outcomes of other laboratories (Kubová et al. 2001). Some authors ascribe the higher horizontal exploratory activity to anxiolytic-like effect of kainic acid (Mikulecká et al. 1999). Such hypothesis seems to be very probable, because rearing duration (sign of exploration) was significantly higher in second half of the test session. Interestingly, one week after the treatment there were no differences between KA treated animals and controls in any of measured parameter, except the weight gain. The finding that effect of low KA dose on animal's exploratory activity and behavior is not long-lasting is new. In histological part of this study we focused our attention on structures of limbic system, because hippocampus is crucial structure for seizure generation (Spencer 2002). No visible signs of neuronal degeneration were observed as confirmed by Fluro-Jade B staining - it leads us to conclusion, that changes observed in behavioral pattern of animals treated with KA would be rather more functional. To conclude, our data showed that animals treated with subconvulsive dose of KA had higher exploratory activity, which starts to be significant thirty minutes after KA treatment. Spontaneous behavior of KA treated animals was substantially modified. Such effect completely disappeared and one week after the treatment locomotion duration, rearing duration returned back to the level of controls. Such changes are very probably not caused by direct neurotoxic damage, because histological analysis did not detected any signs of neuronal damage.

\section{Conflict of Interest}

There is no conflict of interest.

\section{Acknowledgements}

This study was supported by grant by PRVOUKP34/LF1/7.

\section{References}

ALBALA BJ, MOSHE SL, OKADA R: Kainic-acid-induced seizures: a developmental study. Brain Res 315: 139-148, 1984.

DOBLE A: The role of excitotoxicity in neurodegenerative disease: implications for therapy. Pharmacol Ther 81: 163221, 1999.

DREXEL M, PREIDT AP, SPERK G: Sequel of spontaneous seizures after kainic acid-induced status epilepticus and associated neuropathological changes in the subiculum and entorhinal cortex. Neuropharmacology 63: 806$881,2012$.

JANDOVÁ K, MAREŠOVÁ D, POKORNÝ J: Fast and delayed locomotor response to acute high-dose nicotine administration in adult male rats. Physiol Res 62 (Suppl 1): S81-S88, 2013.

KORYNTOVÁ H, MAREŠ P: Effect of a subconvulsant dose of kainic acid on thresholds for phenomena elicited by electrical stimulation of sensorimotor cortex in rats. Epilepsy Res 31: 135-141, 1998.

KUBOVÁ H, MIKULECKÁ A, HAUGVICOVÁ R, LANGMEIER M, MAREŠ P: Nonconvulsive seizures result in behavioral but not electrophysiological changes in developing rats. Epilepsy Behav 2: 473-480, 2001.

MIKULECKÁ A, HLIŇÁK Z, MAREŠ P: Behavioural effects of a subconvulsive dose of kainic acid in rats. Behav Brain Res 101: 21-28, 1999.

MONAGHAN DT, COTMAN CW: The distribution of [3H]kainic acid binding sites in rat CNS as determined by autoradiography. Brain Res 252: 91-100, 1982.

OLNEY JW, RHEE V, HO OL: Kainic acid: a powerful neurotoxic analogue of glutamate. Brain Res 77: 507-512, 1974.

RILJAK V, MILOTOVÁ M, JANDOVÁ K, POKORNÝ J, LANGMEIER M: Morphological changes in the hippocampus following nicotine and kainic acid administration. Physiol Res 56: 641-649, 2007. 
SCHWARZ M, JANDOVÁ K, STRUK I, MAREŠOVÁ D, POKORNÝ J, RILJAK V: Low dose domoic acid influences spontaneous behavior in adult rats. Physiol Res 63: 369-376, 2014.

SPENCER SS: Neural networks in human epilepsy: evidence of and implications for treatment. Epilepsia 43: 219-227, 2002.

SPERK G: Kainic acid seizures in the rat. Prog Neurobiol 42: 1-32, 1994. 